\title{
The $S$-parameter in Holographic Technicolor Models
}

\author{
Kaustubh Agashe ${ }^{a}$, Csaba Csáki ${ }^{b}$, Christophe Grojean ${ }^{c, d}$, and Matthew Reece ${ }^{b}$ \\ ${ }^{a}$ Department of Physics, Syracuse University, Syracuse, NY 13244, USA \\ ${ }^{b}$ Institute for High Energy Phenomenology \\ Newman Laboratory of Elementary Particle Physics, \\ Cornell University, Ithaca, NY 14853, USA \\ ${ }^{d}$ CERN, Theory Division, CH 1211, Geneva 23, Switzerland \\ c Service de Physique Théorique, CEA Saclay, F91191, Gif-sur-Yvette, France \\ kagashe@phy.syr.edu, csaki@lepp.cornell.edu, \\ christophe.grojean@cern.ch, mreece@lepp.cornell.edu
}

\begin{abstract}
We study the $S$ parameter, considering especially its sign, in models of electroweak symmetry breaking (EWSB) in extra dimensions, with fermions localized near the UV brane. Such models are conjectured to be dual to $4 D$ strong dynamics triggering EWSB. The motivation for such a study is that a negative value of $S$ can significantly ameliorate the constraints from electroweak precision data on these models, allowing lower mass scales ( $\mathrm{TeV}$ or below) for the new particles and leading to easier discovery at the LHC. We first extend an earlier proof of $S>0$ for EWSB by boundary conditions in arbitrary metric to the case of general kinetic functions for the gauge fields or arbitrary kinetic mixing. We then consider EWSB in the bulk by a Higgs VEV showing that $S$ is positive for arbitrary metric and Higgs profile, assuming that the effects from higher-dimensional operators in the $5 D$ theory are sub-leading and can therefore be neglected. For the specific case of $\mathrm{AdS}_{5}$ with a power law Higgs profile, we also show that $S \sim+O(1)$, including effects of possible kinetic mixing from higher-dimensional operator (of NDA size) in the $5 D$ theory. Therefore, our work strongly suggests that $S$ is positive in calculable models in extra dimensions.
\end{abstract}




\section{Introduction}

One of the outstanding problems in particle physics is to understand the mechanism of electroweak symmetry breaking. Broadly speaking, models of natural electroweak symmetry breaking rely either on supersymmetry or on new strong dynamics at some scale near the electroweak scale. However, it has long been appreciated that if the new strong dynamics is QCD-like, it is in conflict with precision tests of electroweak observables [1]. Of particular concern is the $S$ parameter. It does not violate custodial symmetry; rather, it is directly sensitive to the breaking of $\mathrm{SU}(2)$. As such, it is difficult to construct models that have $S$ consistent with data, without fine-tuning.

The search for a technicolor model consistent with data, then, must turn to non-QCDlike dynamics. An example is "walking" [2], that is, approximately conformal dynamics, which can arise in theories with extra flavors. It has been argued that such nearly-conformal dynamics can give rise to a suppressed or even negative contribution to the $S$ parameter [3]. However, lacking nonperturbative calculational tools, it is difficult to estimate $S$ in a given technicolor theory.

In recent years, a different avenue of studying dynamical EWSB models has opened up via the realization that extra dimensional models [4] may provide a weakly coupled dual description to technicolor type theories [5]. The most studied of these higgsless models [6] is based on an $\mathrm{AdS}_{5}$ background in which the Higgs is localized on the TeV brane and has a very large VEV, effectively decoupling from the physics. Unitarization is accomplished by gauge KK modes, but this leads to a tension: these KK modes cannot be too heavy or perturbative unitarity is lost, but if they are too light then there are difficulties with electroweak precision: in particular, $S$ is large and positive [7]. In this argument the fermions are assumed to be elementary in the $4 D$ picture (dual to them being localized on the Planck brane). A possible way out is to assume that the direct contribution of the EWSB dynamics to the $S$-parameter are compensated by contributions to the fermion-gauge boson vertices $[8,9]$. In particular, there exists a scenario where the fermions are partially composite in which $S \approx 0$ [10], corresponding to almost flat wave functions for the fermions along the extra dimension. The price of this cancellation is a percent level tuning in the Lagrangian parameter determining the shape of the fermion wave functions. Aside from the tuning itself, this is also undesirable because it gives the model-builder very little freedom in addressing flavor problems: the fermion profiles are almost completely fixed by consistency with electroweak precision.

While Higgsless models are the closest extra-dimensional models to traditional technicolor models, models with a light Higgs in the spectrum do not require light gauge KK modes for unitarization and can be thought of as composite Higgs models. Particularly appealing are those where the Higgs is a pseudo-Nambu-Goldstone boson [11,12]. In these models, the electroweak constraints are less strong, simply because most of the new particles are heavy. They still have a positive $S$, but it can be small enough to be consistent with data. Unlike the Higgsless models where one is forced to delocalize the fermions, in these models with a higher scale the fermions can be peaked near the UV brane so that flavor issues can be addressed.

Recently, an interesting alternative direction to eliminating the $S$-parameter constraint 
has been proposed in [13]. There it was argued, that by considering holographic models of EWSB in more general backgrounds with non-trivial profiles of a bulk Higgs field one could achieve $S<0$. The aim of this paper is to investigate the feasibility of this proposal. We will focus on the direct contribution of the strong dynamics to $S$. In particular, we imagine that the SM fermions can be almost completely elementary in the $4 D$ dual picture, corresponding to them being localized near the UV brane. In this case, a negative $S$ would offer appealing new prospects for model-building since such values of $S$ are less constrained by data than a positive value [14]. Unfortunately we find that the $S>0$ quite generally, and that backgrounds giving negative $S$ appear to be pathological.

The outline of the paper is as follows. We first present a general plausibility argument based purely on 4D considerations that one is unlikely to find models where $S<0$. This argument is independent from the rest of the paper, and the readers interested in the holographic considerations may skip directly to section 3. Here we first review the formalism to calculate the $S$ parameter in quite general models of EWSB using an extra dimension. We also extend the proof of $S>0$ for BC breaking [7] in arbitrary metric to the case of arbitrary kinetic functions or localized kinetic mixing terms. These proofs quite clearly show that no form of boundary condition breaking will result in $S<0$. However, one may hope that (as argued in [13]) one can significantly modify this result by using a bulk Higgs with a profile peaked towards the IR brane to break the electroweak symmetry. Thus, in the crucial section 4, we show that $S>0$ for models with bulk breaking from a scalar VEV as well. Since the gauge boson mass is the lowest dimensional operator sensitive to EWSB one would expect that this is already sufficient to cover all interesting possibilities. However, since the Higgs VEV can be very strongly peaked, one may wonder if other (higher dimensional) operators could become important as well. In particular, the kinetic mixing operator of $L, R$ after Higgs VEV insertion would be a direct contribution to $S$. To study the effect of this operator in section 5, it is shown that the bulk mass term for axial field can be converted to kinetic functions as well, making a unified treatment of the effects of bulk mass terms and the effects of the kinetic mixing from the higher-dimensional operator possible. Although we do not have a general proof that $S>0$ including the effects of the bulk kinetic mixing for a general metric and Higgs profile, in section 5.2 we present a detailed scan for AdS metric and for power-law Higgs vev profile using the technique of the previous section for arbitrary kinetic mixings. We find $S>0$ once we require that the higher-dimensional operator is of NDA size, and that the theory is ghost-free. We summarize and conclude in section 6 .

\section{A plausibility argument for $S>0$}

In this section we define $S$ and sketch a brief argument for its positivity in a general technicolor model. The reader mainly interested in the extra-dimensional constructions can skip this section since it is independent from the rest of the paper. However, we think it is worthwhile to try to understand why one might expect $S>0$ on simple physical grounds. The only assumptions we will make are that we have some strongly coupled theory that spontaneously breaks $\mathrm{SU}(2)_{L} \times \mathrm{SU}(2)_{R}$ down to $\mathrm{SU}(2)_{V}$, and that at high energies the symmetry is 
restored. With these assumptions, $S>0$ is plausible. $S<0$ would require more complicated dynamics, and might well be impossible, though we cannot prove it 1

Consider a strongly-interacting theory with $\mathrm{SU}(2)$ vector current $V_{\mu}^{a}$ and $\mathrm{SU}(2)$ axial vector current $A_{\mu}^{a}$. We define (where $J$ represents $V$ or $A$ ):

$$
i \int d^{4} x e^{-i q \cdot x}\left\langle J_{\mu}^{a}(x) J_{\mu}^{b}(0)\right\rangle=\delta^{a b}\left(q_{\mu} q_{\nu}-g_{\mu \nu} q^{2}\right) \Pi_{J}\left(q^{2}\right) .
$$

We further define the left-right correlator, denoted simply $\Pi\left(q^{2}\right)$, as $\Pi_{V}\left(q^{2}\right)-\Pi_{A}\left(q^{2}\right)$. In the usual way, $\Pi_{V}$ and $\Pi_{A}$ are related to positive spectral functions $\rho_{V}(s)$ and $\rho_{A}(s)$. Namely, the $\Pi$ functions are analytic functions of $q^{2}$ everywhere in the complex plane except for Minkowskian momenta, where poles and branch points can appear corresponding to physical particles and multi-particle thresholds. The discontinuity across the singularities on the $q^{2}>0$ axis is given by a spectral function. In particular, there is a dispersion relation

$$
\Pi_{V}\left(q^{2}\right)=\frac{1}{\pi} \int_{0}^{\infty} d s \frac{\rho_{V}(s)}{s-q^{2}+i \epsilon},
$$

with $\rho_{V}(s)>0$, and similarly for $\Pi_{A}$.

Chiral symmetry breaking establishes that $\rho_{A}(s)$ contains a term $\pi f_{\pi}^{2} \delta(s)$. This is the massless particle pole corresponding to the Goldstone of the spontaneously broken $\mathrm{SU}(2)$ axial flavor symmetry. (The corresponding pions, of course, are eaten once we couple the theory to the Standard Model, becoming the longitudinal components of the $W^{ \pm}$and $Z$ bosons. However, for now we consider the technicolor sector decoupled from the Standard Model.) We define a subtracted correlator by $\bar{\Pi}\left(q^{2}\right)=\Pi\left(q^{2}\right)+\frac{f_{\pi}^{2}}{q^{2}}$ and a subtracted spectral function by $\bar{\rho}_{A}(s)=\rho_{A}(s)-\pi f_{\pi}^{2} \delta(s)$. Now, the $S$ parameter is given by

$$
S=4 \pi \bar{\Pi}(0)=4 \int_{0}^{\infty} d s \frac{1}{s}\left(\rho_{V}(s)-\bar{\rho}_{A}(s)\right) .
$$

Interestingly, there are multiple well-established nonperturbative facts about $\Pi_{V}-\Pi_{A}$, but none are sufficient to prove that $S>0$. There are the famous Weinberg sum rules [17]

$$
\begin{aligned}
\frac{1}{\pi} \int_{0}^{\infty} d s\left(\rho_{V}(s)-\bar{\rho}_{A}(s)\right) & =f_{\pi}^{2}, \\
\frac{1}{\pi} \int_{0}^{\infty} d s s\left(\rho_{V}(s)-\bar{\rho}_{A}(s)\right) & =0 .
\end{aligned}
$$

Further, Witten proved that $\Sigma\left(Q^{2}\right)=-Q^{2}\left(\Pi_{V}\left(Q^{2}\right)-\Pi_{A}\left(Q^{2}\right)\right)>0$ for all Euclidean momenta $Q^{2}=-q^{2}>0$ [18]. However, the positivity of $S$ seems to be more difficult to prove.

Our plausibility argument is based on the function $\Sigma\left(Q^{2}\right)$. In terms of this function, $S=-4 \pi \Sigma^{\prime}(0)$. (Note that in $\Sigma\left(Q^{2}\right)$ the $1 / Q^{2}$ pole from $\Pi_{A}$ is multiplied by $Q^{2}$, yielding a constant that does not contribute when we take the derivative. Thus when considering

\footnotetext{
${ }^{1}$ For a related discussion of the calculation of $S$ in strongly coupled theories, see [15].
} 
$\Sigma$ we do not need to subtract the pion pole as we did in $\bar{\Pi}$.) We also know that $\Sigma(0)=$ $f_{\pi}^{2}>0$. On the other hand, we know something else that is very general about theories that spontaneously break chiral symmetry: at very large Euclidean $Q^{2}$, we should see symmetry restoration. More specifically, we expect behavior like

$$
\Sigma\left(Q^{2}\right) \rightarrow \mathcal{O}\left(\frac{1}{Q^{2 k}}\right)
$$

where $k$ is associated with the dimension of some operator that serves as an order parameter for the symmetry breaking. (In some $5 \mathrm{D}$ models the decrease of $\Pi_{A}-\Pi_{V}$ will actually be faster, e.g. in Higgsless models one has exponential decrease.) While we are most familiar with this from the OPE of QCD, it should be very general. If a theory did not have this property and $\Pi_{V}$ and $\Pi_{A}$ differed significantly in the UV, we would not view it as a spontaneously broken symmetry, but as an explicitly broken one. Now, in this context, positivity of $S$ is just the statement that, because $\Sigma\left(Q^{2}\right)$ begins at a positive value and eventually becomes very small, the smoothest behavior one can imagine is that it simply decreases monotonically, and in particular, that $\Sigma^{\prime}(0)<0$ so that $S>0.2$ The alternative would be that the chiral symmetry breaking effects push $\Sigma\left(Q^{2}\right)$ in different directions over different ranges of $Q^{2}$. We have not proved that this is impossible in arbitrary theories, but it seems plausible that the simpler case is true, namely that chiral symmetry restoration always acts to decrease $\Sigma\left(Q^{2}\right)$ as we move to larger $Q^{2}$. Indeed, we will show below that in a wide variety of perturbative holographic theories $S$ is positive.

\section{Boundary-effective-action approach to oblique cor- rections. Simple cases with boundary breaking}

In this section we review the existing results and calculational methods for the electroweak precision observables (and in particular the $S$-parameter) in holographic models of electroweak symmetry breaking. There are two equivalent formalisms for calculating these parameters. One is using the on-shell wave function of the $W / Z$ bosons [19], and the electroweak observables are calculated from integrals over the extra dimension involving these wave functions. The advantage of this method is that since it uses the physical wave functions it is easier to find connections to the $Z$ and the KK mass scales. The alternative formalism proposed by Barbieri, Pomarol and Rattazzi [7] (and later extended in [20] to include observables off the $Z$-pole) uses the method of the boundary effective action [21], and involves off-shell wave functions of the boundary fields extended into the bulk. This latter method leads more directly to a general expression of the electroweak parameters, so we will be applying this method throughout this paper. Below we will review the basic expressions from [7].

A theory of electroweak symmetry breaking with custodial symmetry has an $\mathrm{SU}(2)_{L} \times$ $\mathrm{SU}(2)_{R}$ global symmetry, of which the $\mathrm{SU}(2)_{L} \times \mathrm{U}(1)_{Y}$ subgroup is gauged (since the $S$ parameter is unaffected by the extra $B-L$ factor we will ignore it in our discussion). At

\footnotetext{
${ }^{2}$ For a related discussion of the behaviour of $\Sigma\left(Q^{2}\right)$ in the case of large- $N_{c}$ QCD, see [16].
} 
low energies, the global symmetry is broken to $\mathrm{SU}(2)_{D}$. In the holographic picture of [7] the elementary $\mathrm{SU}(2) \times \mathrm{U}(1)$ gauge fields are extended into the bulk of the extra dimension. The bulk wave functions are determined by solving the bulk EOM's as a function of the boundary fields, and the effective action is just the bulk action in terms of the boundary fields.

In order to first keep the discussion as general as possible, we use an arbitrary background metric over an extra dimension parametrized by $0<y<1$, where $y=0$ corresponds to the UV boundary, and $y=1$ to the IR boundary. In order to simplify the bulk equations of motion it is preferential to use the coordinates in which the metric takes the form 11 [7]

$$
d s^{2}=e^{2 \sigma} d x^{2}+e^{4 \sigma} d y^{2}
$$

The bulk action for the gauge fields is given by

$$
\mathcal{S}=-\frac{1}{4 g_{5}^{2}} \int d^{5} x \sqrt{-g}\left(\left(F_{M N}^{L}\right)^{2}+\left(F_{M N}^{R}\right)^{2}\right)
$$

The bulk equations of motion are given by

$$
\partial_{y}^{2} A_{\mu}^{L, R}-p^{2} e^{2 \sigma} A_{\mu}^{L, R}=0,
$$

or equivalently the same equations for the combinations $V_{\mu}, A_{\mu}=\left(A_{\mu L} \pm A_{\mu R}\right) / \sqrt{2}$.

We assume that the (light) SM fermions are effectively localized on the Planck brane and that they carry their usual quantum numbers under $S U(2)_{L} \times U(1)_{Y}$ that remains unbroken on the UV brane. The values of these fields on the UV brane have therefore a standard couplings to fermion and they are the 4D interpolating fields we want to compute an effective action for. This dictates the boundary conditions we want to impose on the UV brane

$$
A_{\mu}^{L a}\left(p^{2}, 0\right)=\bar{A}_{\mu}^{L a}\left(p^{2}\right), A_{\mu}^{R 3}\left(p^{2}, 0\right)=\bar{A}_{\mu}^{R 3}\left(p^{2}\right), A_{\mu}^{R 1,2}\left(p^{2}, 0\right)=0 .
$$

$A_{R}^{1,2}$ are vanishing because they correspond to ungauged symmetry generators. The solutions of the bulk equations of motion satisfying these UV BC's take the form

$$
V_{\mu}\left(p^{2}, y\right)=v\left(y, p^{2}\right) \bar{V}_{\mu}\left(p^{2}\right), A_{\mu}\left(p^{2}, y\right)=a\left(y, p^{2}\right) \bar{A}_{\mu}\left(p^{2}\right) .
$$

where the interpolating functions $v$ and $a$ satisfy the bulk equations

$$
\partial_{y}^{2} f\left(y, p^{2}\right)-p^{2} e^{2 \sigma} f\left(y, p^{2}\right)=0
$$

and the UV BC's

$$
v\left(0, p^{2}\right)=1, a\left(0, p^{2}\right)=1
$$

The effective action for the boundary fields reduces to a pure boundary term since by integrating by parts the bulk action vanishes by the EOM's:

$$
\mathcal{S}_{\text {eff }}=\left.\frac{1}{2 g_{5}^{2}} \int d^{4} x\left(V_{\mu} \partial_{y} V^{\mu}+A_{\mu} \partial_{y} A^{\mu}\right)\right|_{y=0}=\left.\frac{1}{2 g_{5}^{2}} \int d^{4} p\left(\bar{V}_{\mu}^{2} \partial_{y} v+\bar{A}_{\mu}^{2} \partial_{y} a\right)\right|_{y=0}
$$

\footnotetext{
${ }^{1}$ In this paper, we use a $(-+\ldots+)$ signature. 5D bulk indices are denoted by capital Latin indices while we use Greek letters for 4D spacetime indices. 5D indices will be raised and lowered using the 5D metric while the 4D Minkowski metric is used for 4D indices.
} 
And we obtain the non-trivial vacuum polarizations for the boundary vector fields

$$
\Sigma_{V}\left(p^{2}\right)=-\frac{1}{g_{5}^{2}} \partial_{y} v\left(0, p^{2}\right), \Sigma_{A}\left(p^{2}\right)=-\frac{1}{g_{5}^{2}} \partial_{y} a\left(0, p^{2}\right) .
$$

The various oblique electroweak parameters are then obtained from the momentum expansion of the vacuum polarizations in the effective action,

$$
\Sigma\left(p^{2}\right)=\Sigma(0)+p^{2} \Sigma^{\prime}(0)+\frac{p^{4}}{2} \Sigma^{\prime \prime}(0)+\ldots
$$

For example the $S$-parameter is given by

$$
S=16 \pi \Sigma_{3 B}^{\prime}(0)=8 \pi\left(\Sigma_{V}^{\prime}(0)-\Sigma_{A}^{\prime}(0)\right)
$$

A similar momentum expansion can be performed on the interpolating functions $v$ and $a$ : $v\left(y, p^{2}\right)=v^{(0)}(y)+p^{2} v^{(1)}(y)+\ldots$, and similarly for $a$. The $S$-parameter is then simply expressed as

$$
S=-\left.\frac{8 \pi}{g_{5}^{2}}\left(\partial_{y} v^{(1)}-\partial_{y} a^{(1)}\right)\right|_{y=0}
$$

The first general theorem was proved in [7]: for the case of boundary condition breaking in a general metric, $S \geq 0$. The proof uses the explicit calculation of the functions $v^{(n)}, a^{(n)}, n=$ 0,1 . First, the bulk equations (3.3) write

$$
\partial_{y}^{2} v^{(0)}=\partial_{y}^{2} a^{(0)}=0, \partial_{y}^{2} v^{(1)}=e^{2 \sigma} v^{(0)}, \partial_{y}^{2} a^{(1)}=e^{2 \sigma} a^{(0)} .
$$

And the $p^{2}$-expanded UV BC's are

$$
v^{(0)}=a^{(0)}=1, v^{(1)}=a^{(1)}=0 \text { at } y=0
$$

Finally, we need to specify the BC's on the IR brane that correspond to the breaking $S U(2)_{L} \times \mathrm{SU}(2)_{R} \rightarrow \mathrm{SU}(2)_{D}$

$$
\partial_{y} V_{\mu}=0, A_{\mu}=0
$$

which translates into simple BC's for the interpolating functions

$$
\partial_{y} v^{(n)}=a^{(n)}=0, n=0,1 .
$$

The solution of these equations are $v^{(0)}=1, a^{(0)}=1-y, v^{(1)}=\int_{0}^{y} d y^{\prime} \int_{0}^{y^{\prime}} d y^{\prime \prime} e^{2 \sigma\left(y^{\prime \prime}\right)}-$ $y \int_{0}^{1} d y^{\prime} e^{2 \sigma\left(y^{\prime}\right)}, a^{(1)}=\int_{0}^{y} d y^{\prime} \int_{0}^{y^{\prime}} d y^{\prime \prime} e^{2 \sigma\left(y^{\prime \prime}\right)}\left(1-y^{\prime \prime}\right)-y \int_{0}^{1} d y^{\prime} \int_{0}^{y^{\prime}} d y^{\prime \prime} e^{2 \sigma\left(y^{\prime \prime}\right)}\left(1-y^{\prime \prime}\right)$. Consequently

$$
S=\frac{8 \pi}{g_{5}^{2}}\left(\int_{0}^{1} d y e^{2 \sigma(y)} d y-\int_{0}^{1} d y \int_{0}^{y} d y^{\prime}\left(1-y^{\prime}\right) e^{2 \sigma\left(y^{\prime}\right)}\right)
$$

which is manifestly positive. 


\section{1 $S>0$ for BC breaking with boundary kinetic mixing}

The first simple generalization of the BC breaking model is to consider the same model but with an additional localized kinetic mixing operator added on the $\mathrm{TeV}$ brane (the effect of this operator has been studied in flat space in [7] and in AdS space in [19]). The localized Lagrangian is

$$
-\frac{\tau}{4 g_{5}^{2}} \int d^{4} x \sqrt{-g} V_{\mu \nu}^{2}
$$

This contains only the kinetic term for the vector field since the axial gauge field is set to zero by the $\mathrm{BC}$ breaking. In this case the $\mathrm{BC}$ at $y=1$ for the vector field is modified to $\partial_{y} V_{\mu}+\tau p^{2} V_{\mu}=0$. In terms of the wave functions expanded in small momenta we get $\partial_{y} v^{(1)}+\tau v^{(0)}=0$. The only change in the solutions will be that now $v^{(1)^{\prime}}=-\tau-\int_{y}^{1} e^{2 \sigma\left(y^{\prime}\right)} d y^{\prime}$, resulting in

$$
S=\frac{8 \pi}{g_{5}^{2}}\left(\int_{0}^{1} e^{2 \sigma(y)} d y-\int_{0}^{1} d y \int_{0}^{y}\left(1-y^{\prime}\right) e^{2 \sigma\left(y^{\prime}\right)} d y^{\prime}+\tau\right)
$$

Thus as long as the localized kinetic term has the proper sign, the shift in the $S$-parameter will be positive. If the sign is negative, there will be an instability in the theory since fields localized very close to the $\mathrm{TeV}$ brane will feel a wrong sign kinetic term. Thus we conclude that for the physically relevant case $S$ remains positive.

\section{2 $S>0$ for BC breaking with arbitrary kinetic functions}

The next simple extension of the BPR result is to consider the case when there is an arbitrary $y$-dependent function in front of the bulk gauge kinetic terms. These could be interpreted as effects of gluon condensates modifying the kinetic terms in the IR. In this case the action is

$$
\mathcal{S}=-\frac{1}{4 g_{5}^{2}} \int d^{5} x \sqrt{-g}\left(\phi_{L}^{2}(y)\left(F_{M N}^{L}\right)^{2}+\phi_{R}^{2}(y)\left(F_{M N}^{R}\right)^{2}\right) .
$$

$\phi_{L, R}(y)$ are arbitrary profiles for the gauge kinetic terms, which are assumed to be the consequence of some bulk scalar field coupling to the gauge fields. Note that this case also covers the situation when the gauge couplings are constant but $g_{5 L} \neq g_{5 R}$. The only assumption we are making is that the gauge kinetic functions for $L, R$ are strictly positive. Otherwise one could create a wave packet localized around the region where the kinetic term is negative which would have ghost-like behavior.

Due to the $y$-dependent kinetic terms it is not very useful to go into the $V, A$ basis. Instead we will directly solve the bulk equations in the original basis. The bulk equations of motion for $L, R$ are given by

$$
\partial_{y}\left(\phi_{L, R}^{2} \partial_{y} A_{\mu}^{L, R}\right)-p^{2} e^{2 \sigma} \phi_{L, R}^{2} A_{\mu}^{L, R}=0
$$

To find the boundary effective action needed to evaluate the S-parameter we perform the following decomposition:

$$
\begin{aligned}
& A_{\mu}^{L}\left(p^{2}, y\right)=\bar{L}_{\mu}\left(p^{2}\right) L_{L}\left(y, p^{2}\right)+\bar{R}_{\mu}\left(p^{2}\right) L_{R}\left(y, p^{2}\right) \\
& A_{\mu}^{R}\left(p^{2}, y\right)=\bar{L}_{\mu}\left(p^{2}\right) R_{L}\left(y, p^{2}\right)+\bar{R}_{\mu}\left(p^{2}\right) R_{R}\left(y, p^{2}\right) .
\end{aligned}
$$


Here $\bar{L}, \bar{R}$ are the boundary fields, and the fact that we have four wave functions expresses the fact that these fields will be mixing due to the BC's on the IR brane. The UV BC's (3.4) and the IR BC's (3.15) can be written in terms of the interpolating functions as

$$
\begin{array}{ll}
(\mathrm{UV}) & L_{L}\left(0, p^{2}\right)=1, L_{R}\left(0, p^{2}\right)=0, R_{L}\left(0, p^{2}\right)=0, R_{R}\left(0, p^{2}\right)=1 . \\
& L_{L}\left(1, p^{2}\right)=R_{L}\left(1, p^{2}\right), L_{R}\left(1, p^{2}\right)=R_{R}\left(1, p^{2}\right), \\
& \partial_{y}\left(L_{L}\left(1, p^{2}\right)+R_{L}\left(1, p^{2}\right)\right)=0, \partial_{y}\left(L_{R}\left(1, p^{2}\right)+R_{R}\left(1, p^{2}\right)\right)=0 .
\end{array}
$$

The solution of these equations with the proper boundary conditions and for small values of $p^{2}$ is rather lengthy, so we have placed the details in Appendix $\mathrm{A}$. The end result is that

$$
S=-\left.\frac{8 \pi}{g_{5}^{2}}\left(\phi_{L}^{2} \partial_{y} L_{R}^{(1)}+\phi_{R}^{2} \partial_{y} R_{L}^{(1)}\right)\right|_{y=0}=-\frac{8 \pi}{g_{5}^{2}}\left(a_{L_{R}}+a_{R_{L}}\right)
$$

where the constants $a_{R_{L}}$ are negative as their explicit expressions shows it. Therefore $S$ is positive.

\section{$4 \quad S>0$ in models with bulk Higgs}

Having shown than $S>0$ for arbitrary metric and EWSB through BC's, in this section, we switch to considering breaking of electroweak symmetry by a bulk scalar (Higgs) vev. We begin by neglecting the effects of kinetic mixing between $S U(2)_{L}$ and $S U(2)_{R}$ fields coming from higher-dimensional operator in the $5 D$ theory, expecting that their effect, being suppressed by the $5 D$ cut-off, is sub-leading. We will return to a consideration of such kinetic mixing effects in the following sections.

We will again use the metric (3.1) and the bulk action (3.2). Instead of $\mathrm{BC}$ breaking we assume that EWSB is caused by a bulk Higgs which results in a $y$-dependent profile for the axial mass term

$$
-\int d^{5} x \sqrt{-g} \frac{M^{2}(y)}{2 g_{5}^{2}} A_{M}^{2}
$$

Here $M^{2}$ is a positive function of $y$ corresponding to the background Higgs VEV. The bulk equations of motion are:

$$
\left(\partial_{y}^{2}-p^{2} e^{2 \sigma}\right) V_{\mu}=0, \quad\left(\partial_{y}^{2}-p^{2} e^{2 \sigma}-M^{2} e^{4 \sigma}\right) A_{\mu}=0 .
$$

On the IR brane, we want to impose regular Neumann BC's that preserve the full $\mathrm{SU}(2)_{L} \times$ $\mathrm{SU}(2)_{R}$ gauge symmetry

$$
(I R) \quad \partial_{y} V_{\mu}=0, \quad \partial_{y} A_{\mu}=0
$$

As in the previous section, the BC's on the UV brane just define the $4 \mathrm{D}$ interpolating fields

$$
(U V) V_{\mu}\left(p^{2}, 0\right)=\bar{V}_{\mu}\left(p^{2}\right), \quad A_{\mu}\left(p^{2}, 0\right)=\bar{A}_{\mu}\left(p^{2}\right) .
$$


The solutions of the bulk equations of motion satisfying these BC's take the form

$$
V_{\mu}\left(p^{2}, y\right)=v\left(y, p^{2}\right) \bar{V}_{\mu}\left(p^{2}\right), \quad A_{\mu}\left(p^{2}, y\right)=a\left(y, p^{2}\right) \bar{A}_{\mu}\left(p^{2}\right)
$$

where the interpolating functions $v$ and $a$ satisfy the bulk equations

$$
\partial_{y}^{2} v-p^{2} e^{2 \sigma} v=0, \quad \partial_{y}^{2} a-p^{2} e^{2 \sigma} a-M^{2} e^{4 \sigma} a=0 .
$$

As before, these interpolating functions are expanded in powers of the momentum: $v\left(y, p^{2}\right)=$ $v^{(0)}(y)+p^{2} v^{(1)}(y)+\ldots$, and similarly for $a$. The $S$-parameter is again given by the same expression

$$
S=-\left.\frac{8 \pi}{g_{5}^{2}}\left(\partial_{y} v^{(1)}-\partial_{y} a^{(1)}\right)\right|_{y=0} .
$$

We will not be able to find general solutions for $a^{(1)}$ and $v^{(1)}$ but we are going to prove that $\partial_{y} a^{(1)}>\partial_{y} v^{(1)}$ on the UV brane, which is exactly what is needed to conclude that $S>0$.

First at the zeroth order in $p^{2}$, the solution for $v^{(0)}$ is simply constant, $v^{(0)}=1$, as before. And $a^{(0)}$ is the solution of

$$
\partial_{y}^{2} a^{(0)}=M^{2} e^{4 \sigma} a^{(0)},\left.\quad a^{(0)}\right|_{y=0}=1,\left.\quad \partial_{y} a^{(0)}\right|_{y=1}=0 .
$$

In particular, since $a^{(0)}$ is positive at $y=0$, this implies that $a^{(0)}$ remains positive: if $a^{(0)}$ crosses through zero it must be decreasing, but then this equation shows that the derivative will continue to decrease and can not become zero to satisfy the other boundary condition. Now, since $a^{(0)}$ is positive, the equation of motion shows that it is always concave up, and then the condition that its derivative is zero at $y=1$ shows that it is a decreasing function of $y$. In particular, we have for all $y$

$$
a^{(0)}(y) \leq v^{(0)}(y)
$$

with equality only at $y=0$.

Next consider the order $p^{2}$ terms. What we wish to show is that $\partial_{y} a^{(1)}>\partial_{y} v^{(1)}$ at the UV brane. First, let's examine the behavior of $v^{(1)}$ : the boundary conditions are $\left.v^{(1)}\right|_{y=0}=0$ and $\left.\partial_{y} v^{(1)}\right|_{y=1}=0$. The equation of motion is:

$$
\partial_{y}^{2} v^{(1)}=e^{2 \sigma} v^{(0)}=e^{2 \sigma}>0
$$

so the derivative of $v^{(1)}$ must increase to reach zero at $y=1$. Thus it is negative everywhere except $y=1$, and $v^{(1)}$ is a monotonically decreasing function of $y$. Since $\left.v^{(1)}\right|_{y=0}=0, v^{(1)}$ is strictly negative on $(0,1]$.

For the moment suppose that $a^{(1)}$ is also strictly negative; we will provide an argument for this shortly. The equation of motion for $a^{(1)}$ is:

$$
\partial_{y}^{2} a^{(1)}=e^{2 \sigma} a^{(0)}+M^{2} e^{4 \sigma} a^{(1)} .
$$

Now, we know that $a^{(0)}<v^{(0)}$, so under our assumption that $a^{(1)}<0$, this means that

$$
\partial_{y}^{2} a^{(1)} \leq \partial_{y}^{2} v^{(1)}
$$


with equality only at $y=0$. But we also know that $\partial_{y} v^{(1)} \partial_{y} a^{(1)}$ at $y=1$, since they both satisfy Neumann boundary conditions there. Since the derivative of $\partial_{y} a^{(1)}$ is strictly smaller over $(0,1]$, it must start out at a higher value in order to reach the same boundary condition. Thus we have that

$$
\left.\partial_{y} a^{(1)}\right|_{y=0}>\left.\partial_{y} v^{(1)}\right|_{y=0} .
$$

The assumption that we made is that $a^{(1)}$ is strictly negative over the interval $(0,1]$. The reason is the following: suppose that $a^{(1)}$ becomes positive at some value of $y$. Then as it passes through zero it is increasing. But then we also have that $\partial_{y}^{2} a^{(1)}=e^{2 \sigma} a^{(0)}+M^{2} e^{4 \sigma} a^{(1)}$, and we have argued above that $a^{(0)}>0$. Thus if $a^{(1)}$ is positive, $\partial_{y} a^{(1)}$ remains positive, because $\partial_{y}^{2} a^{(1)}$ cannot become negative. In particular, it becomes impossible to reach the boundary condition $\partial_{y} a^{(1)}=0$ at $y=1$. This fills the missing step in our argument and shows that the $S$ parameter must be positive.

In the rest of this section we show that the above proof for the positivity of $\mathrm{S}$ remains essentially unchanged in the case when the bulk gauge couplings for the $\mathrm{SU}(2)_{L}$ and $\mathrm{SU}(2)_{R}$ gauge groups are not equal. In this case (in order to get diagonal bulk equations of motion) one needs to also introduce the canonically normalized gauge fields. We start with the generic action (metric factors are understood when contracting indices)

$$
\int d^{5} x \sqrt{-g}\left(-\frac{1}{4 g_{5 L}^{2}}\left(F_{M N}^{L}\right)^{2}-\frac{1}{4 g_{5 R}^{2}}\left(F_{M N}^{R}\right)^{2}-\frac{h^{2}(z)}{2}\left(L_{M}-R_{M}\right)^{2}\right)
$$

To get to a canonically normalized diagonal basis we redefine the fields as

$$
\tilde{A}=\frac{1}{\sqrt{g_{5 L}^{2}+g_{5 R}^{2}}}(L-R), \tilde{V}=\frac{1}{\sqrt{g_{5 L}^{2}+g_{5 R}^{2}}}\left(\frac{g_{5 R}}{g_{5 L}} L+\frac{g_{5 L}}{g_{5 R}} R\right) .
$$

To get the boundary effective action, we write the fields $\tilde{V}, \tilde{A}$ as

$$
\begin{aligned}
\tilde{A}\left(p^{2}, z\right) & =\frac{1}{\sqrt{g_{5 L}^{2}+g_{5 R}^{2}}}\left(\bar{L}\left(p^{2}\right)-\bar{R}\left(p^{2}\right)\right) \tilde{a}\left(p^{2}, z\right) \\
\tilde{V}\left(p^{2}, z\right) & =\frac{1}{\sqrt{g_{5 L}^{2}+g_{5 R}^{2}}}\left(\frac{g_{5 R}}{g_{5 L}} \bar{L}\left(p^{2}\right)+\frac{g_{5 L}}{g_{5 R}} \bar{R}\left(p^{2}\right)\right) \tilde{v}\left(p^{2}, z\right) .
\end{aligned}
$$

Here $\bar{L}, \bar{R}$ are the boundary effective fields (with non-canonical normalization exactly as in [7]), while the profiles $\tilde{a}, \tilde{v}$ satisfy the same bulk equations and boundary conditions as $a, v$ in (4.2)-(4.4) with an appropriate replacement for $M^{2}=\left(g_{5 L}^{2}+g_{5 R}^{2}\right) h^{2}$. In terms of the canonically normalized fields, the boundary effective action takes its usual form

$$
\mathcal{S}_{\text {eff }}=\frac{1}{2} \int d^{4} x\left(\tilde{V} \partial_{y} \tilde{V}+\tilde{A} \partial_{y} \tilde{A}\right)_{y=0} .
$$

And we deduce the vacuum polarization

$$
\Sigma_{L 3 B}\left(p^{2}\right)=-\frac{1}{g_{5 L}^{2}+g_{5 R}^{2}}\left(\partial_{y} \tilde{v}\left(0, p^{2}\right)-\partial_{y} \tilde{a}\left(0, p^{2}\right)\right)
$$


And finally the $S$-parameter is equal to

$$
S=-\frac{16 \pi}{g_{5 L}^{2}+g_{5 R}^{2}}\left(\partial_{y} \tilde{v}^{(1)}-\partial_{y} \tilde{a}^{(1)}\right)
$$

Since $\tilde{a}^{(n)}, \tilde{v}^{(n)}, n=0,1$ satisfy the same equations (4.2)-(4.4) as before, the proof goes through unchanged and we conclude that $S>0$.

\section{$5 \quad$ Bulk Higgs and bulk kinetic mixing}

Next, we wish to consider the effects of kinetic mixing from higher-dimensional operator in the bulk involving the Higgs VEV - as mentioned earlier, this kinetic mixing is suppressed by the $5 D$ cut-off and hence expected to be a sub-leading effect. The reader might wonder why we neglected it before, but consider it now? The point is that, although the leading effect on $S$ parameter is positive as shown above, it can be accidentally suppressed so that the formally sub-leading effects from the bulk kinetic mixing can be important, in particular, such effects could change the sign of $S$. Also, the Higgs VEV can be large, especially when the Higgs profile is "narrow" such that it approximates BC breaking, and thus the large VEV can (at least partially) compensate the suppression from the $5 D$ cut-off. Of course, in this limit of BC breaking ( $\delta$-function $\mathrm{VEV}$ ), we know that kinetic mixing gives $S<0$ only if tachyons are present in the spectrum, but we would like to cover the cases intermediate between BC breaking limit and a broad Higgs profile as well. In this section, we develop a formalism, valid for arbitrary metric and Higgs profile, to treat the bulk mass term and kinetic mixing on the same footing and then we apply this technique to models in AdS space and with power-law profiles for Higgs VEV in the next section.

We first present a discussion of how a profile for the $y$-dependent kinetic term is equivalent to a bulk mass term. This is equivalent to the result [13] that a bulk mass term can be equivalent to an effective metric. However, we find the particular formulation that we present here to be more useful when we deal with the case of a kinetic mixing. Assume we have a Lagrangian for a gauge field that has a kinetic term

$$
\mathcal{S}=-\frac{1}{4 g_{5}^{2}} \int d^{5} x \sqrt{-g} \phi^{2}(y) F_{M N}^{2}
$$

We work in the axial gauge $A_{5}=0$ and again the metric takes the form (3.1). We redefine the field to absorb the function $\phi: \tilde{A}(y)=\phi(y) A(y)$. The action in terms of the new field is then written as

$$
\mathcal{S}=-\frac{1}{4 g_{5}^{2}} \int d^{5} x\left(e^{2 \sigma} \tilde{F}_{\mu \nu}^{2}+2\left(\partial_{y} \tilde{A}_{\mu}\right)^{2}+2 \frac{\phi^{\prime 2}}{\phi^{2}} \tilde{A}_{\mu}^{2}-4\left(\partial_{y} \tilde{A}_{\mu}\right) \tilde{A}^{\mu} \frac{\phi^{\prime}}{\phi}\right)
$$

To see that the kinetic profile $\phi$ is equivalent to a mass term, we integrate by parts in the second term

$$
\mathcal{S}=-\frac{1}{4 g_{5}^{2}} \int d^{5} x \sqrt{-g}\left(\tilde{F}_{M N}^{2}+2 e^{-4 \sigma} \frac{\phi^{\prime \prime}}{\phi} \tilde{A}_{\mu}^{2}\right)+\left.\frac{1}{2 g_{5}^{2}} \int d^{4} x \frac{\phi^{\prime}}{\phi} \tilde{A}_{\mu}^{2}\right|_{0} ^{1}
$$


Thus we find that a bulk kinetic profile is equivalent to a bulk mass plus a boundary mass. The bulk equations of motion for the new variables will then be

$$
\partial_{y}^{2} \tilde{A}_{\mu}-e^{2 \sigma} p^{2} \tilde{A}_{\mu}-\frac{\phi^{\prime \prime}}{\phi} \tilde{A}_{\mu}=0
$$

and the boundary conditions become

$$
\partial_{y} \tilde{A}_{\mu}=\frac{\phi^{\prime}}{\phi} \tilde{A}_{\mu}
$$

Note, that despite the bulk mass term, there is still a massless mode whose wavefunction is simply $\phi(z)$. Now we can reverse the argument and say that a bulk mass must be equivalent to a profile for the bulk kinetic term plus a boundary mass term.

\subsection{The general case}

We have seen above how to go between a bulk mass terms and a kinetic function. We will now use this method to discuss the general case, when there is electroweak symmetry breaking due to a bulk higgs with a sharply peaked profile toward the IR brane, and the same Higgs introduces kinetic mixing between L and R fields corresponding to a higher dimensional operator from the bulk. For now we assume that the Higgs fields that breaks the electroweak symmetry is in a $(2,2)$ of $\mathrm{SU}(2)_{L} \times \mathrm{SU}(2)_{R}$, with a $\operatorname{VEV}\langle H\rangle=\operatorname{diag}(h(z), h(z)) / \sqrt{2} 11$ This Higgs profile $h$ has dimension $3 / 2$. The $5 \mathrm{D}$ action is given by

$$
\int d^{5} x \sqrt{-g}\left[-\frac{1}{4 g_{5}^{2}}\left[\left(F_{M N}^{L}\right)^{2}+\left(F_{M N}^{R}\right)^{2}\right]-\left(D_{M} H\right)^{\dagger}\left(D^{M} H\right)+\frac{\alpha}{\Lambda^{2}} \operatorname{Tr}\left(F_{M N}^{L} H^{\dagger} H F^{M N} R\right)\right] .
$$

Here $\alpha$ is a coefficient of $\mathcal{O}(1)$ and $\Lambda$ is the $5 \mathrm{D}$ cutoff scale, given approximately by $\Lambda \sim$ $24 \pi^{3} / g_{5}^{2}$. The kinetic mixing term just generates a shift in the kinetic terms of the vector and axial vector field, and we will write the bulk mass term also as a shift in the kinetic term for the axial vector field. The exact form of the translation between the two forms is given by answering the question of how to redefine the field with an action (note that $\mathrm{m}^{2}$ has a mass dimension 3)

$$
-\frac{1}{4 g_{5}^{2}} \int d^{5} x \sqrt{-g}\left(w F_{M N}^{2}+m^{2} 2 g_{5}^{2} A_{\mu} A^{\mu}\right)
$$

to a theory with only a modified kinetic term. The appropriate field redefinition $A=\rho \tilde{A}$ will be canceling the mass term if $\rho$ satisfies

$$
\partial_{y}\left(w \partial_{y} \rho\right)=m^{2} g_{5}^{2} e^{4 \sigma} \rho
$$

\footnotetext{
${ }^{1}$ An alternative possibility would be to consider a Higgs in the $(3,3)$ representation of $\mathrm{SU}(2)_{L} \times \mathrm{SU}(2)_{R}$.
} 
together with the boundary conditions $\left.\rho^{\prime}\right|_{y=1}=0,\left.\rho\right|_{y=0}=1$. The relation between the new and the old expression for $w$ will be $\tilde{w}=\rho^{2} w$. The action in this case is given by

$$
-\frac{1}{4 g_{5}^{2}} \int d^{5} x \sqrt{-g} \tilde{w}_{M N}^{2}+\left.\int d^{4} x \frac{\tilde{w}(0)}{2 g_{5}^{2}}\left(\partial_{y} \rho\right) \tilde{A}^{2}\right|_{y=0}
$$

This last boundary term is actually irrelevant for the $S$-parameter: since it does not contain a derivative on the field it can not get an explicit $p$-dependence so it will not contribute to $S$, so for practical purposes this boundary term can be neglected.

With this expression we now can calculate $S$. For this we need the modified version of the formula from [13], where the breaking is not by boundary conditions but by a bulk Higgs. The expression is

$$
S=\frac{8 \pi}{g_{5}^{2}} \int_{0}^{1} d y e^{2 \sigma}\left(w_{V}-\tilde{w}_{A}\right)
$$

In our case $w_{V}=1-\frac{\alpha h^{2}(y) 2 g_{5}^{2}}{\Lambda^{2}}$ while $\tilde{w}_{A}=w_{A} \rho^{2}=\left(1+\frac{\alpha h^{2}(y) 2 g_{5}^{2}}{\Lambda^{2}}\right) \rho^{2}$.

This formula also gives another way to see that $S>0$ in the absence of kinetic mixing, without analyzing the functions $v^{(1)}$ and $a^{(1)}$ from Section 4 in detail. Without kinetic mixing, $w_{V}=1$ and $\tilde{w}_{A}=\rho^{2}$, and the equation of motion for $\rho$ is simply $\partial_{y}^{2} \rho=m^{2} g_{5}^{2} e^{4 \sigma} \rho$. In that case $\rho$ is just the function we called $a^{(0)}$ in Section 4 . Since we showed there that $a^{(0)} \leq 1$, we see that our expression 5.10 gives an alternative argument that $S>0$ without kinetic mixing, because it is simply an integral of $e^{2 \sigma}\left(1-\rho^{2}\right) \geq 0$.

\subsection{Scan of the parameter space for AdS backgrounds}

Having developed the formalism for a unified treatment of bulk mass terms and bulk kinetic mixing, we then apply it to the AdS case with a power-law profile for the Higgs vev. Requiring (i) calculability of the 5D theory, i.e., NDA size of the higher-dimensional operator, (ii) that excited $W / Z$ 's are heavier than a few $100 \mathrm{GeV}$, and (iii) a ghost-free theory, i.e., positive kinetic terms for both $V$ and $A$ fields, we find that $S$ is always positive in our scan for this model. We do not have a general proof that $S>0$ for an arbitrary background with arbitrary Higgs profiles, if we include the effects of the bulk kinetic mixing, but we feel that such a possibility is quite unlikely based on our exhaustive scan. For this scan we will take the parametrization of the Higgs profile from [22]. Here the metric is taken as AdS space

$$
d s^{2}=\left(\frac{R}{z}\right)^{2}\left(\eta_{\mu \nu} d x^{\mu} d x^{\nu}-d z^{2}\right),
$$

where as usual $R<z<R^{\prime}$. The bulk Higgs VEV is assumed to be a pure monomial in $z$ (rather than a combination of an increasing and a decreasing function). The reason for this is that we are only interested in the effect of the strong dynamics on the electroweak precision parameters. A term in the Higgs VEV growing toward the UV brane would mean that the value of bulk Higgs field evaluated on the UV brane gets a VEV, implying that there is EWSB also by a elementary Higgs (in addition to the strong dynamics) in the $4 D$ 
dual. We do not want to consider such a case. The form of the Higgs VEV is then assumed to be

$$
v(z)=\sqrt{\frac{2(1+\beta) \log R^{\prime} / R}{\left(1-\left(R / R^{\prime}\right)^{2+2 \beta}\right)}} \frac{g V}{g_{5}} \frac{R^{\prime}}{R}\left(\frac{z}{R^{\prime}}\right)^{2+\beta},
$$

where the parameter $\beta$ characterizes how peaked the Higgs profile is toward the TeV brane ( $\beta \rightarrow-1$ corresponds to a flat profile, $\beta \rightarrow \infty$ to an infinitely peaked one). The other parameter $V$ corresponds to an "effective Higgs VEV", and is normalized such that for $V \rightarrow 246 \mathrm{GeV}$ we recover the $\mathrm{SM}$ and the $\mathrm{KK}$ modes decouple $\left(R^{\prime} \rightarrow \infty\right.$ irrespective of $\left.\beta\right)$. For more details about the definitions of these parameters see [22].2

We first numerically fix the $R^{\prime}$ parameter for every given $V, \beta$ and kinetic mixing parameter $\alpha$ by requiring that the $W$-mass is reproduced. We do this approximately, since we assume the simple matching relation $1 / g^{2}=R \log \left(R^{\prime} / R\right) / g_{5}^{2}$ to numerically fix the value of $g_{5}$, which is only true to leading order, but due to wave function distortions and the extra kinetic term will get corrected. Then, $\rho$ can be numerically calculated by solving (5.8), and from this $S$ can be obtained via (5.10).

We see that $S$ decreases as we increase $\alpha$. On the the hand, the kinetic function for vector field $\left(w_{V}\right)$ also decreases in this limit. So, in order to find the minimal value of $S$ consistent with the absence of ghosts in the theory, we find numerically the maximal value of $\alpha$ for every value of $V, \beta$ for which the kinetic function of the vectorlike gauge field is still strictly positive. We then show contour plots for the minimal value of $S$ taking this optimal value of $\alpha$ as a function of $V, \beta$ in Fig. 5.2. In the first figure we fix $R^{\prime}=10^{-8} \mathrm{GeV}^{-1}$, which is the usual choice for higgsless models with light KK W' and Z' states capable of rendering the model perturbative. In the second plot we choose the more conventional value $R=10^{-18}$ $\mathrm{GeV}^{-1}$. We can see the $S$ is positive in both cases over all of the physical parameter space.

We can estimate the corrections to the above matching relation from the wavefunction distortion and kinetic mixing as follows. The effect from wavefunction distortion is expected to be $\sim g^{2} S /(16 \pi)$ which is $\lesssim 10 \%$ if we restrict to regions of parameter space with $S \lesssim 10$. Similarly, we estimate the effect due to kinetic mixing by simply integrating the operator over the extra dimension to find a deviation $\sim g^{6}\left(V R^{\prime}\right)^{2} \log ^{2}\left(R^{\prime} / R\right) /\left(24 \pi^{3}\right)^{2}$. So, if restrict to $V \lesssim 1 \mathrm{TeV}$ and $1 / R^{\prime} \gtrsim 100 \mathrm{GeV}$, then this deviation is also small enough. We see that both effects are small due to the deviation being non-zero only near IR brane - even though it is $\mathrm{O}(1)$ in that region, whereas the zero-mode profile used in the matching relation is spread throughout the extra dimension.

In order to be able to make a more general statement (and to check that the neglected additional contributions to the gauge coupling matching from the wave function distortions and the kinetic mixing indeed do not significantly our results) we have performed an additional scan over AdS space models where we do not require the correct physical value of $M_{W}$ to be reproduced. In this scan we then treat $R^{\prime}$ as an independent free parameter. In this case the correct matching between $g$ and $g_{5}$ is no longer important for the sign of $S$, since at

\footnotetext{
${ }^{2}$ Refs. [23] also considered similar models.
} 

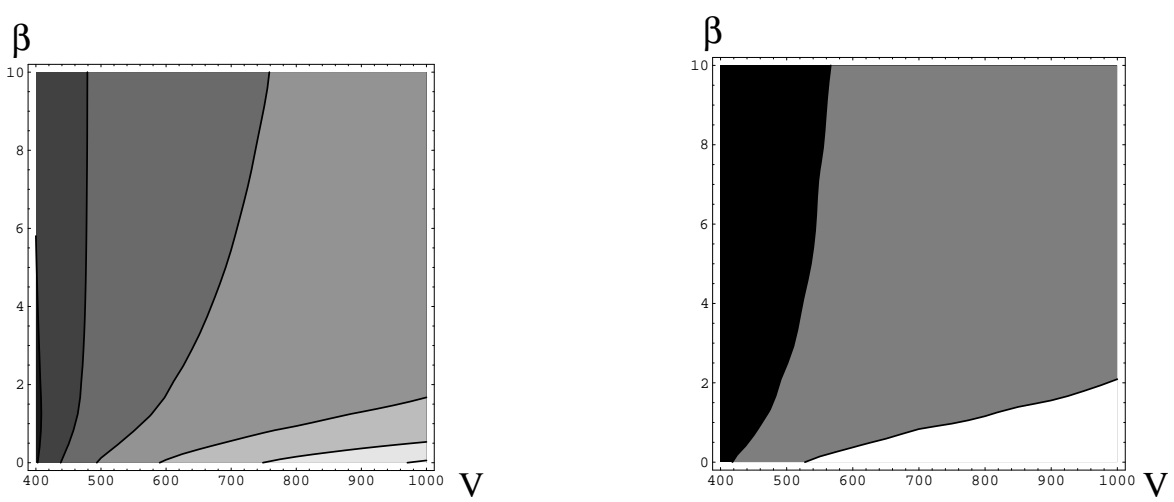

Figure 1: The contours of models with fixed values of the $S$-parameter due to the electroweak breaking sector. In the left panel we fix $1 / R=10^{8} \mathrm{GeV}$, while in the right $1 / R=10^{18} \mathrm{GeV}$. The gauge kinetic mixing parameter $\alpha$ is fixed to be the maximal value corresponding to the given $V, \beta$ (and $R^{\prime}$ chosen such that the $\mathrm{W}$ mass is approximately reproduced). In the left panel the contours are $S=1,2,3,4,5,6$, while in the right $S=1,1.5,2$.

every place where $g_{5}$ appears it is multiplied by a parameter we are scanning over anyway $(V$ or $\alpha)$.

We performed the scan again for two values of the AdS curvature, $1 / R=10^{8}$ and $10^{18}$ $\mathrm{GeV}$. For the first case we find that if we restrict $\alpha<10,1 / R^{\prime}<1 \mathrm{TeV}$ there is no case with $S<0$. However, there are some cases with $S<0$ for $\alpha>10$, although in these cases the theory is likely not predictive. For $1 / R=10^{18} \mathrm{GeV}$ we find that $S<0$ only for $V \sim 250$ $\mathrm{GeV}$ and $\beta \sim 0,1 / R^{\prime} \sim 1 \mathrm{TeV}$. In this case $\alpha$ is of order one (for example $\alpha \sim 5$ ). This case corresponds to the composite Higgs model of [11] and it is quite plausible that at tree-level $S<0$ if a large kinetic mixing is added in the bulk. However in this case EWSB is mostly due to a Higgs, albeit a composite particle of the strong dynamics, rather than directly by the strong dynamics, so it does not contradict the expectation that when EWSB is triggered directly via strong dynamics, then $\mathrm{S}$ is always large and positive. However, it shows that any general proof for $S>0$ purely based on analyzing the properties of Eqs. (5.8)-(5.10) is doomed to failure, since these equations contain physical situations where EWSB is not due to the strong dynamics but due to a light Higgs in the spectrum. Thus any general proof likely needs to include more physical requirements on the decoupling of the physical Higgs.

\section{Conclusions}

In this paper, we have studied the $S$ parameter in holographic technicolor models, focusing especially on its sign. The motivation for our study was as follows. An alternative (to SUSY) solution to the Planck-weak hierarchy involves a strongly interacting $4 D$ sector spontaneously breaking the EW symmetry. One possibility for such a strong sector is a scaled-up version of QCD as in the traditional technicolor models. In such models, we can use the QCD data to "calculate" $S$ finding $S \sim+O(1)$ which is ruled out by the electroweak precision 
data. Faced by this constraint, the idea of a "walking" dynamics was proposed and it can be then argued that $S<0$ is possible which is much less constrained by the data, but the $S$ parameter cannot be calculated in such models. In short, there is a dearth of calculable models of (non-supersymmetric) strong dynamics in $4 D$.

Based on the AdS/CFT duality, the conjecture is that certain kinds of theories of strong dynamics in $4 D$ are dual to physics of extra dimensions. The idea then is to construct models of EWSB in an extra dimension. Such constructions allow more possibilities for model-building, at the same time maintaining calculability if the $5 D$ strong coupling scale is larger than the compactification scale, corresponding to large number of technicolors in the $4 D$ dual.

It was already shown that $S>0$ for boundary condition breaking for arbitrary metric (a proof for $S>0$ for the case of breaking by a localized Higgs vev was recently studied in reference [24]). In this paper, we have extended the proof for boundary condition breaking to the case of arbitrary bulk kinetic functions for gauge fields or gauge kinetic mixing.

Throughout this paper, we have assumed that the (light) SM fermions are effectively localized near the UV brane so that flavor violation due to higher-dimensional operators in the $5 D$ theory can be suppressed, at the same time allowing for a solution to the flavor hierarchy. Such a localization of the light SM fermions in the extra dimension is dual to SM fermions being "elementary", i.e., not mixing with composites from the $4 D$ strong sector. It is known that the $S$ parameter can be suppressed (or even switch sign) for a flat profile for SM fermions (or near the TeV brane) - corresponding to mixing of elementary fermions with composites in the $4 D$ dual, but in such a scenario flavor issues could be a problem.

We also considered the case of bulk breaking of the EW symmetry motivated by recent arguments that $S<0$ is possible with different effective metrics for vector and axial fields. For arbitrary metric and Higgs profile, we showed that $S>0$ at leading order, i.e., neglecting effects from all higher-dimensional operators in the $5 D$ theory (especially bulk kinetic mixing), which are expected to be sub-leading effects being suppressed by the cut-off of the $5 D$ theory. We also note that boundary mass terms can generally be mimicked to arbitrary precision by localized contributions to the bulk scalar profile, so we do not expect a more general analysis of boundary plus bulk breaking to find new features. Obtaining $S<0$ must then require either an unphysical Higgs profile or higher-dimensional operators to contribute effects larger than NDA size, in which case we lose calculability of the $5 D$ theory.

To make our case for $S>0$ stronger, we then explored effects of the bulk kinetic mixing between $S U(2)_{L, R}$ gauge fields due to Higgs vev coming from a higher-dimensional operator in the $5 D$ theory. Even though, as mentioned above, this effect is expected to be sub-leading, it can nevertheless be important (especially for the sign of $S$ ) if the leading contribution to $S$ is accidentally suppressed. Also, the large Higgs VEV, allowed for narrow profiles in the extra dimension (approaching the BC breaking limit), can compensate the suppression due to the cut-off in this operator. For this analysis, we found it convenient to convert bulk (mass) ${ }^{2}$ for gauge fields also to kinetic functions. Although a general proof for $S>0$ is lacking in such a scenario, using the above method of treating the bulk mass for axial fields, we found that $S \sim+O(1)$ for $\mathrm{AdS}_{5}$ model with power-law Higgs profile in the viable (ghost-free) and calculable regions of the parameter space. 
In summary, our results combined with the previous literature strongly suggests that $S$ is positive for calculable models of technicolor in $4 D$ and $5 D$. We also presented a plausibility argument for $S>0$ which is valid in general, i.e., even for non-calculable models.

\section{Acknowledgments}

We thank Giacomo Cacciapaglia, Cédric Delaunay, Antonio Delgado, Guido Marandella, Riccardo Rattazzi, Matthew Schwartz and Raman Sundrum for discussions. We also thank Johannes Hirn and Veronica Sanz for comments on the manuscript. As we were finishing the paper, we learned that Raman Sundrum and Tom Kramer have also obtained results similar to ours [25]. C.C. thanks the theory group members at CERN for their hospitality during his visit. K.A. is supported in part by the U. S. DOE under Contract no. DE-FG-02-85ER 40231. The research of C.C. is supported in part by the DOE OJI grant DE-FG02-01ER41206 and in part by the NSF grant PHY-0355005. The research of M.R. is supported in part by an NSF graduate student fellowship and in part by the NSF grant PHY-0355005. C.G. is supported in part by the RTN European Program MRTN-CT-2004-503369 and by the CNRS/USA exchange grant 3503.

\section{Note added}

After submitting our paper to the arXiv, we learned of [26] which gives a proof for $S>0$ for an arbitrary bulk Higgs profile in AdS background that is similar to our proof in Section 4. However, our proof of $S>0$ is valid for a general metric and we have also included the effect of kinetic mixing between $S U(2)_{L}$ and $S U(2)_{R}$ fields via higher-dimensional operator (with Higgs vev) for the calculation of $S$ in AdS background. We thank Deog-Ki Hong and Ho-Ung Yee for pointing out their paper to us.

\section{A Details of BC breaking with arbitrary kinetic func- tions}

Here we present the detailed calculation of $S$ in the case with boundary breaking and arbitrary kinetic functions described in Section 3.2 . Recall that we had the following decomposition:

$$
\begin{aligned}
& A_{\mu}^{L}\left(p^{2}, y\right)=\bar{L}_{\mu}\left(p^{2}\right) L_{L}\left(y, p^{2}\right)+\bar{R}_{\mu}\left(p^{2}\right) L_{R}\left(y, p^{2}\right) \\
& A_{\mu}^{R}\left(p^{2}, y\right)=\bar{L}_{\mu}\left(p^{2}\right) R_{L}\left(y, p^{2}\right)+\bar{R}_{\mu}\left(p^{2}\right) R_{R}\left(y, p^{2}\right)
\end{aligned}
$$


with boundary conditions

$$
\begin{array}{ll}
(\mathrm{UV}) & L_{L}\left(0, p^{2}\right)=1, L_{R}\left(0, p^{2}\right)=0, R_{L}\left(0, p^{2}\right)=0, R_{R}\left(0, p^{2}\right)=1 . \\
(\mathrm{IR}) & L_{L}\left(1, p^{2}\right)=R_{L}\left(1, p^{2}\right), L_{R}\left(1, p^{2}\right)=R_{R}\left(1, p^{2}\right) \\
& \partial_{y}\left(L_{L}\left(1, p^{2}\right)+R_{L}\left(1, p^{2}\right)\right)=0, \partial_{y}\left(L_{R}\left(1, p^{2}\right)+R_{R}\left(1, p^{2}\right)\right)=0 .
\end{array}
$$

The action again reduces to a boundary term

$$
\mathcal{S}_{\text {eff }}=\frac{1}{2 g_{5}^{2}}\left(\phi_{L}^{2}(0) L_{\mu} \partial L^{\mu}+\phi_{R}^{2}(0) R_{\mu} \partial R^{\mu}\right)
$$

so we find that

$$
S=-\left.\frac{8 \pi}{g_{5}^{2}}\left(\phi_{L}^{2} \partial_{y} L_{R}^{(1)}+\phi_{R}^{2} \partial_{y} R_{L}^{(1)}\right)\right|_{y=0}
$$

where we have done an expansion in terms of the momentum for all the wave functions as usual as $L_{L}\left(y, p^{2}\right)=L_{L}^{(0)}(y)+p^{2} L_{L}^{(1)}(y)+\ldots$. The lowest order wave functions satisfy the following bulk equations:

$$
\partial_{y}\left(\phi_{I}^{2} \partial_{y} I_{J}^{(0)}\right)=0
$$

where $I$ and $J$ can refer to $L$ or $R$. Imposing the BC's these equations can be simply solved in terms of the integrals

$$
f_{L}(y)=\frac{\int_{0}^{y} \frac{d y^{\prime}}{\phi_{L}^{2}\left(y^{\prime}\right)}}{\int_{0}^{1} \frac{d y^{\prime}}{\phi_{L}^{2}\left(y^{\prime}\right)}+\frac{\phi_{R}^{2}(1)}{\phi_{L}^{2}(1)} \int_{0}^{1} \frac{d y^{\prime}}{\phi_{R}^{2}\left(y^{\prime}\right)}}, f_{R}(y)=\frac{\int_{0}^{y} \frac{d y^{\prime}}{\phi_{R}^{2}\left(y^{\prime}\right)}}{\int_{0}^{1} \frac{d y^{\prime}}{\phi_{R}^{2}\left(y^{\prime}\right)}+\frac{\phi_{L}^{2}(1)}{\phi_{R}^{2}(1)} \int_{0}^{1} \frac{d y^{\prime}}{\phi_{L}^{2}\left(y^{\prime}\right)}}
$$

as

$$
L_{L}^{(0)}=1-f_{L}(y), L_{R}^{(0)}=f_{L}(y), R_{L}^{(0)}=f_{R}(y), R_{R}^{(0)}=1-f_{R}(y)
$$

In order to actually find $\mathrm{S}$ we need to go one step further, that is calculate the next order terms in the wave functions $I_{J}^{(1)}$. These will satisfy the equations

$$
\partial_{y}\left(\phi_{I}^{2} \partial_{y} I_{J}^{(1)}\right)=e^{2 \sigma} \phi_{I}^{2} I_{J}^{(0)}
$$

where for the $I_{J}^{(0)}$ we use the solutions in (A.8). The form of the solutions will be given by

$$
I_{J}^{(1)}(y)=\int_{0}^{y} \frac{d y^{\prime}}{\phi_{I}^{2}\left(y^{\prime}\right)} \int_{0}^{y^{\prime}} d u e^{2 \sigma} \phi_{I}^{2}(u) I_{J}^{(0)}(u)+a_{I_{J}} \int_{0}^{y} \frac{d y^{\prime}}{\phi_{I}^{2}}
$$

where $a_{I_{J}}$ are constants. In terms of these quantities the S-parameter is just given by

$$
S=-\frac{8 \pi}{g_{5}^{2}}\left(a_{L_{R}}+a_{R_{L}}\right)
$$


One can again solve the boundary conditions to find the constants $a_{R_{L}}, a_{L_{R}}$. These turn out to be

$$
\begin{aligned}
& a_{R_{L}}=-\frac{1}{N_{R}}\left[\int_{0}^{1} \frac{d y}{\phi_{L}^{2}(y)} \int_{y}^{1} d y^{\prime} e^{2 \sigma\left(y^{\prime}\right)} \phi_{L}^{2}\left(y^{\prime}\right)\left(1-f_{L}\left(y^{\prime}\right)\right)\right. \\
& \left.+\frac{\phi_{L}^{2}(1)}{\phi_{R}^{2}(1)} \int_{0}^{1} d y e^{2 \sigma(y)} \phi_{R}^{2}(y) f_{R}(y) \int_{0}^{1} \frac{d y}{\phi_{L}^{2}(y)}+\int_{0}^{1} \frac{d y}{\phi_{R}^{2}(y)} \int_{0}^{y} d y^{\prime} e^{2 \sigma\left(y^{\prime}\right)} \phi_{R}^{2}\left(y^{\prime}\right) f_{R}\left(y^{\prime}\right)\right]
\end{aligned}
$$

where

$$
N_{R}=\int_{0}^{1} \frac{d y}{\phi_{R}^{2}(y)}+\frac{\phi_{L}^{2}(1)}{\phi_{R}^{2}(1)} \int_{0}^{1} \frac{1}{\phi_{L}^{2}(y)} .
$$

A similar expressions applies for $a_{L_{R}}$ with $L \leftrightarrow R$ everywhere. Since $0<f_{L, R}<1$, we can see that every term in the expression is manifestly positive, so $S$ is definitely positive.

\section{References}

[1] M. E. Peskin and T. Takeuchi, Phys. Rev. Lett. 65, 964 (1990); B. Holdom and J. Terning, Phys. Lett. B 247, 88 (1990); M. Golden and L. Randall, Nucl. Phys. B 361, 3 (1991); M. E. Peskin and T. Takeuchi, Phys. Rev. D 46, 381 (1992).

[2] B. Holdom, Phys. Rev. D 24, 1441 (1981).

[3] R. Sundrum and S. D. H. Hsu, Nucl. Phys. B 391, 127 (1993) arXiv:hep-ph/9206225; T. Appelquist and F. Sannino, Phys. Rev. D 59, 067702 (1999) arXiv:hep-ph/9806409.

[4] L. Randall and R. Sundrum, Phys. Rev. Lett. 83, 3370 (1999) arXiv:hep-ph/9905221.

[5] N. Arkani-Hamed, M. Porrati and L. Randall, JHEP 0108, 017 (2001) arXiv:hep-th/0012148.

[6] C. Csáki, C. Grojean, H. Murayama, L. Pilo and J. Terning, Phys. Rev. D 69, 055006 (2004) |arXiv:hep-ph/0305237]; C. Csáki, C. Grojean, L. Pilo and J. Terning, Phys. Rev. Lett. 92, 101802 (2004) arXiv:hep-ph/0308038.

[7] R. Barbieri, A. Pomarol and R. Rattazzi, Phys. Lett. B 591, 141 (2004) arXiv:hep-ph/0310285.

[8] C. Grojean, W. Skiba and J. Terning, Phys. Rev. D 73, 075008 (2006) arXiv:hep-ph/0602154.

[9] G. Cacciapaglia, C. Csáki, G. Marandella and A. Strumia, Phys. Rev. D 74, 033011 (2006) arXiv:hep-ph/0604111. 
[10] G. Cacciapaglia, C. Csáki, C. Grojean and J. Terning, Phys. Rev. D 71, 035015 (2005) arXiv:hep-ph/0409126]; R. Foadi, S. Gopalakrishna and C. Schmidt, Phys. Lett. B 606, 157 (2005) |arXiv:hep-ph/0409266]; R. S. Chivukula, E. H. Simmons, H. J. He, M. Kurachi and M. Tanabashi, Phys. Rev. D 71, 115001 (2005) arXiv:hep-ph/0502162.

[11] R. Contino, Y. Nomura and A. Pomarol, Nucl. Phys. B 671, 148 (2003) arXiv:hep-ph/0306259]; K. Agashe, R. Contino and A. Pomarol, Nucl. Phys. B 719, 165 (2005) |arXiv:hep-ph/0412089|; K. Agashe and R. Contino, Nucl. Phys. B 742, 59 (2006) arXiv:hep-ph/0510164.

[12] G. F. Giudice, C. Grojean, A. Pomarol and R. Rattazzi, arXiv:hep-ph/0703164.

[13] J. Hirn and V. Sanz, Phys. Rev. Lett. 97, 121803 (2006) arXiv:hep-ph/0606086 ; J. Hirn and V. Sanz, arXiv:hep-ph/0612239.

[14] W. M. Yao et al. [Particle Data Group], J. Phys. G 33, 1 (2006).

[15] M. Kurachi and R. Shrock, Phys. Rev. D 74, 056003 (2006) arXiv:hep-ph/0607231.

[16] S. Friot, D. Greynat and E. de Rafael, JHEP 0410, 043 (2004) arXiv:hep-ph/0408281.

[17] S. Weinberg, Phys. Rev. Lett. 18, 507 (1967).

[18] E. Witten, Phys. Rev. Lett. 51, 2351 (1983).

[19] C. Csáki, J. Erlich and J. Terning, Phys. Rev. D 66, 064021 (2002) arXiv:hep-ph/0203034]; G. Cacciapaglia, C. Csáki, C. Grojean and J. Terning, Phys. Rev. D 70, 075014 (2004) arXiv:hep-ph/0401160|.

[20] R. Barbieri, A. Pomarol, R. Rattazzi and A. Strumia, Nucl. Phys. B 703, 127 (2004) arXiv:hep-ph/0405040.

[21] S. S. Gubser, I. R. Klebanov and A. M. Polyakov, Phys. Lett. B 428, 105 (1998) |arXiv:hep-th/9802109]; E. Witten, Adv. Theor. Math. Phys. 2, 253 (1998) [arXiv:hep-th/9802150].

[22] G. Cacciapaglia, C. Csáki, G. Marandella and J. Terning, arXiv:hep-ph/0611358.

[23] H. Davoudiasl, B. Lillie and T. G. Rizzo, JHEP 0608, 042 (2006) arXiv:hep-ph/0508279]; C. D. Carone, J. Erlich and J. A. Tan, Phys. Rev. D 75, 075005 (2007) |arXiv:hep-ph/0612242]; M. Piai, arXiv:hep-ph/0609104 and arXiv:hep-ph/0608241.

[24] A. Delgado and A. Falkowski, arXiv:hep-ph/0702234.

[25] T. Kramer and R. Sundrum, private communication.

[26] D. K. Hong and H. U. Yee, Phys. Rev. D 74, 015011 (2006) arXiv:hep-ph/0602177. 